\title{
A Microsaccadic Rhythm Modulates Gamma-Band Synchronization and Behavior
}

\author{
Conrado A. Bosman, ${ }^{1}$ Thilo Womelsdorf, ${ }^{1}$ Robert Desimone,,${ }^{2,3}$ and Pascal Fries ${ }^{1}$ \\ ${ }^{1}$ Donders Institute for Brain, Cognition and Behaviour, Radboud University Nijmegen, 6525 EN Nijmegen, The Netherlands, ${ }^{2}$ Laboratory of \\ Neuropsychology, National Institute of Mental Health, National Institutes of Health, Bethesda, Maryland 20892, and ${ }^{3}$ McGovern Institute for Brain \\ Research at the Massachusetts Institute of Technology, Cambridge, Massachusetts 02139
}

\begin{abstract}
Rhythms occur both in neuronal activity and in behavior. Behavioral rhythms abound at frequencies at or below $10 \mathrm{~Hz}$. Neuronal rhythms cover a very wide frequency range, and the phase of neuronal low-frequency rhythms often rhythmically modulates the strength of higher-frequency rhythms, particularly of gamma-band synchronization (GBS). Here, we study stimulus-induced GBS in awake monkey areas V1 and V4 in relation to a specific form of spontaneous behavior, namely microsaccades (MSs), small fixational eye movements. We found that MSs occur rhythmically at a frequency of $\sim 3.3 \mathrm{~Hz}$. The rhythmic MSs were predicted by the phase of the $3.3 \mathrm{~Hz}$ rhythm in V1 and V4 local field potentials. In turn, the MSs modulated both visually induced GBS and the speed of visually triggered behavioral responses. Fast/slow responses were preceded by a specific temporal pattern of MSs. These MS patterns induced perturbations in GBS that in turn explained variability in behavioral response speed. We hypothesize that the $3.3 \mathrm{~Hz}$ rhythm structures the sampling and exploration of the environment through building and breaking neuronal ensembles synchronized in the gamma-frequency band to process sensory stimuli.
\end{abstract}

\section{Introduction}

Rhythms abound both in behavior and in neuronal activity (Singer and Gray, 1995; Lakatos et al., 2005; Buzsáki, 2006). Behavioral and neuronal rhythms come at many different frequencies and the relationship between rhythms of different frequencies has recently received much attention. One prominent and recurring finding is that the phase of lower-frequency rhythms modulates the strength or precision of higher-frequency rhythms. This so-called cross-frequency nested coherence has been described in several species and in several parts of the nervous system (Bragin et al., 1995; Lakatos et al., 2005; Canolty et al., 2006; Osipova et al., 2008; Fries, 2009; Händel and Haarmeier, 2009; Schroeder and Lakatos, 2009).

Recent research has indicated that salient events in one sensory modality can reset the phase of the low-frequency rhythm in other sensory modalities and probably across much of the neocortex (Fanselow and Nicolelis, 1999; Lakatos et al., 2005, 2007, 2008; Rajkai et al., 2008). The reset of the low-frequency phase renders the coherent high-frequency synchronization particularly suitable to process new incoming information. Furthermore, not only sensory stimuli but also spontaneous saccades can

Received March 11, 2009; revised May 14, 2009; accepted June 13, 2009.

This work was supported by Beca Presidente de la República, Gobierno de Chile (C.A.B.), the European Community's Seventh Framework Programme (FP7/2007-2013), Grant Agreement "BrainSynch" HEALTH-F2-2008-200728 (P.F.), The Volkswagen Foundation Grant I/79876 (P.F.), the European Science Foundation European Young Investigator Award Program (P.F.), The Netherlands Organization for Scientific Research Grant 452-03-344 (P.F.), the National Institute of Mental Health Intramural Research Program (R.D.), and National Institute of Health Grant R01-EY017292 (R.D.). We thank J. H. Reynolds, A. E. Rorie, and A. F. Rossi for help during the monkey experiments. This article is freely available online through the J Neurosci Open Choice option.

Correspondence should be addressed to Dr. Conrado Bosman, Donders Institute for Brain, Cognition and Behaviour, Centre of Cognitive Neuroimaging, Radboud University Nijmegen, Kapittelweg 29, 6525 EN Nijmegen, The Netherlands. E-mail: c.bosman@donders.ru.nl.

DOI:10.1523/JNEUROSCI.1193-09.2009

Copyright $\odot 2009$ Society for Neuroscience $\quad 0270-6474 / 09 / 299471-10 \$ 15.00 / 0$ reset low-frequency rhythms and trigger well timed enhancements of neuronal activity that aid the processing of the newly acquired postsaccadic retinal image (Rajkai et al., 2008).

However, we do not yet understand fully the relation of these phenomena to the many forms of rhythmic behavior. Rhythmic behavior is prominent also in sensory systems, in which it typically scans the sensory surface across the environment and thereby constitutes so-called active sensation. Active sensation has received much attention in the rodent vibrissal system, in which the vibrissae are moved across the input space rhythmically (Kleinfeld et al., 2006; Mehta et al., 2007; Lee et al., 2008). Such rhythmic exploration has also been suggested for the visual system and it has been pointed out that the high-frequency rhythmic microtremor of the eyes might constitute a scanning of the visual environment (Ahissar and Arieli, 2001).

As in many systems, several rhythms might well coexist in the oculomotor system and interact with each other. In addition to microtremor, the eyes also display drifts, saccades, and microsaccades (MSs) (Martinez-Conde et al., 2004). Saccades constitute overt exploratory behavior and microsaccades are related to them in many respects (Otero-Millan et al., 2008; Hafed et al., 2009). We therefore analyzed the temporal pattern of microsaccades and found a prominent low-frequency rhythm around $3.3 \mathrm{~Hz}$. Since the low-frequency rhythms often pattern high-frequency rhythms, we analyzed the relation between microsaccades and visually induced gamma-band activity and found strong modulations both in area $\mathrm{V} 1$ and in area V4. Finally, we analyzed the relation between the microsaccadic rhythm, the corresponding rhythmic modulation of visual cortical gamma band activity, and behavior. We found that fast/slow sensorymotor transformations are predicted in part by the phase of the microsaccade rhythm in a way that is 
consistent with the corresponding rhythmic modulation of visual cortical gamma-band activity.

\section{Materials and Methods}

Behavioral paradigm and visual stimulation. Two male macaque monkeys were trained in a change detection paradigm (Fries et al., 2001, 2008b; Womelsdorf et al., 2006, 2007). Trials started when the monkey touched a bar. This turned on a $0.2^{\circ}$ diameter fixation point. When the monkey brought its gaze into a window of $\pm 0.7^{\circ}$ around the fixation point, a prestimulus baseline of $1 \mathrm{~s}$ started. After this baseline period, two stimuli appeared that were in most cases placed in different visual quadrants and in few cases spaced more closely. The stimuli were pure luminance gratings with a contrast of $100 \%$, a diameter of $2-3^{\circ}$, a spatial frequency of $1-2$ cycles ${ }^{\circ}$, and a temporal frequency of $1-2 \%$. They were presented on a monitor screen $57 \mathrm{~cm}$ from the monkey's eyes at a resolution of $800 \times 600$ pixels and a frame rate of $120 \mathrm{~Hz}$. One of the gratings was inside the receptive fields of the recorded neurons, whereas the other was outside. The monkey's attention was directed to one of the stimuli by means of a visual cue. Monkeys were trained to respond to a color change of the cued grating and ignore the noncued one. The analysis presented here focused on the MS-related modulations in neuronal activity and synchronization and therefore pooled data from all correctly performed trials of both attention conditions.

Surgery. Procedures were done in accordance with National Institutes of Health guidelines. Electrophysiological recordings were made in areas V1 and V4 of two monkeys, as described previously (Fries et al., 2001, 2008b; Womelsdorf et al., 2006). Briefly, under aseptic conditions and with isofluorane anesthesia, monkeys were surgically implanted with a head post and with a scleral search coil for the recording of the eye position. Preoperative structural magnetic resonance imaging was conducted to identify the stereotactic coordinates of V1 and V4. In a separate surgery, plastic recording chambers were placed according to the determined coordinates. The skull remained intact during this procedure. Three days before the first recording session, by use of ketamine-xylazine anesthesia, small holes of 3-5 mm diameter were drilled through the skull inside the chamber to expose the dura for electrode penetrations.

Recording techniques. Recordings were obtained from four to eight tungsten electrodes (impedances of 1-2 M $\Omega$ ) positioned in V1 and V4 with a hydraulic microstep multidrive (FHC) and four guide tubes organized in a square arrangement (separation between electrodes: 650-900 $\mu \mathrm{m}$ ). Electrodes were lowered through the dura and into the cortex one by one and at a slow rate $(1.5 \mu \mathrm{m} / \mathrm{s})$ to avoid cortical deformation ("dimpling"). Signal acquisition, filtering, and amplification were done with a multineuron acquisition system (Plexon). Each electrode's signal was passed through a headstage of unit gain and subsequently split to extract the spike and local field potential (LFP) components. For spike recordings, the signals were filtered with a passband of $100-8000 \mathrm{~Hz}$, further amplified, and digitized with $40 \mathrm{kHz}$. A threshold was set interactively and spike waveforms were stored for a time window from $150 \mu$ s before to $700 \mu$ s after threshold crossing. The threshold clearly separated spikes from noise but was chosen to include multiunit activity (MUA). Off-line, we performed a principal component analysis of the waveforms (Offline Sorter, Plexon) and plotted the first against the second principal component. Those waveforms that corresponded to artifacts were excluded. All other waveforms were pooled as multiunit activity. For all further analyses involving spikes, only the times of threshold crossing were kept and downsampled to $1 \mathrm{kHz}$. In V1, multiunit recordings were obtained from 111 sites (79 and 32 in monkeys A and B, respectively) and LFP recordings from 109 sites (77 and 32, respectively). In V4, multiunit recordings were obtained from 61 sites ( 39 and 22 in monkeys A and B, respectively), and LFP recordings from 64 sites (40 and 24, respectively). LFP signals were bandpass filtered at $0.7-170 \mathrm{~Hz}$, amplified, and digitized at $1 \mathrm{kHz}$.

Eye position was monitored using a scleral search coil system (DNI) with one coil implanted in one of the eyes. Horizontal and vertical eye position was digitized at a sampling rate of $1 \mathrm{kHz}$.

Microsaccade detection. MSs were detected according to the following procedure. Horizontal and vertical eye position recordings were low-pass filtered $(<40 \mathrm{~Hz}$ ) to remove high-frequency noise. The filtered position signals were differentiated in time to obtain velocity signals. Horizontal and vertical eye velocities were then combined to give overall eye velocity, regardless of movement direction. If this velocity exceeded 3 SDs of its mean, this was considered a saccade. This threshold was determined separately for each session and corresponded on average (across sessions) to an eye velocity of $4.43 \%$. This value is close to values used in previous studies (Martinez-Conde et al., 2000, 2006). Saccades that stayed within the predefined fixation window of $0.7^{\circ}$ around the fixation point were considered MSs. Of this group, we excluded MSs with amplitudes smaller than the 5th percentile of the amplitude distribution or larger than the 95th percentile. To confirm that the correspondingly selected eye movements were MSs, we additionally tested for the well described linear relationship between peak velocity and amplitude of the MS (Zuber and Stark, 1965).

LFP preprocessing. The powerline artifact was removed from the LFP using a discrete Fourier transform (DFT) filter (Schoffelen et al., 2005; Womelsdorf et al., 2006). All signals had been recorded continuously for the entire duration of the recording session. For each time epoch of interest (and each recording channel), we first took a 10 s epoch out of the continuous signal with the epoch of interest in the middle. We then calculated the DFT of the 10 s epoch at 60,120 , and $180 \mathrm{~Hz}$ without any tapering. Since the powerline artifact is of a perfectly constant frequency, the $10 \mathrm{~s}$ epoch contains integer cycles of the artifact frequencies and all the artifact energy is contained in those DFTs. We then constructed 60, 120 , and $180 \mathrm{~Hz}$ sine waves with the amplitudes and phases as estimated by the respective DFTs and subtracted those sine waves from the $10 \mathrm{~s}$ epoch. The epoch of interest was then cut out of the cleaned $10 \mathrm{~s}$ epoch. Power spectra of the cleaned $10 \mathrm{~s}$ epochs demonstrated that all artifact energy was eliminated, leaving a notch of a bin width of $0.1 \mathrm{~Hz}(=1 / 10 \mathrm{~s})$. The actual spectral analysis used the multitaper method, resulting in an effective spectral (boxcar) smoothing over several hertz (see below for details). Thus, the notch typically became invisible.

Time-frequency spectral analyses. We were interested in the perimicrosaccadic dynamics of visually induced neuronal activation and synchronization. Therefore, we selected all data epochs from correctly performed trials in which the monkey fixated and there was a visual stimulus inside the receptive field of the recorded neurons. We excluded only the first $0.2 \mathrm{~s}$ after stimulus onset to avoid onset transients. We then analyzed neural data within \pm 0.8 s around MSs. Rhythmic neuronal synchronization was assessed with spectra of LFP power and of coherence between spikes and LFP. These spectra were calculated for sliding windows that were moved over the perimicrosaccade epochs in steps of $0.01 \mathrm{~s}$. In timefrequency plots, the time-axis displays the times of the centers of the sliding windows. We used different window lengths and different spectral smoothing for lower and higher frequencies, because of the different bandwidths of the typical lower- and higher-physiological-frequency bands. Frequencies between 5 and $25 \mathrm{~Hz}$ were analyzed with windows of $0.4 \mathrm{~s}$ length and a spectral smoothing of $\pm 3.75 \mathrm{~Hz}$. Frequencies between 25 and $105 \mathrm{~Hz}$ were analyzed with windows of $0.2 \mathrm{~s}$ length and a spectral smoothing of $\pm 7.5 \mathrm{~Hz}$.

Optimized spectral smoothing/concentration was achieved using the multitaper method (Mitra and Pesaran, 1999; Jarvis and Mitra, 2001). For each taper, the data epoch was multiplied with that taper and then Fourier transformed, giving the windowed Fourier transform as follows:

$$
\tilde{x}_{k}(f)=\sum_{1}^{N} w_{k}(t) x_{t} e^{-2 \pi i f t}
$$

where $x_{t},(t=1,2, \ldots, N)$ is the time series of the signal under consideration and $w_{k}(t),(k=1,2, \ldots, K)$ are $K$ orthogonal taper functions.

The multitaper estimates for the (power) spectrum $S_{x}(f)$ and the cross-spectrum $S_{y x}(f)$ are given by

$$
S_{x}(f)=\frac{1}{K} \sum_{1}^{K}\left|\tilde{x}_{k}(f)\right|^{2}
$$

and

$$
S_{y x}(f)=\frac{1}{K} \sum_{1}^{K} \tilde{y}_{k}(f) \tilde{x}_{k}^{*}(f)
$$


A

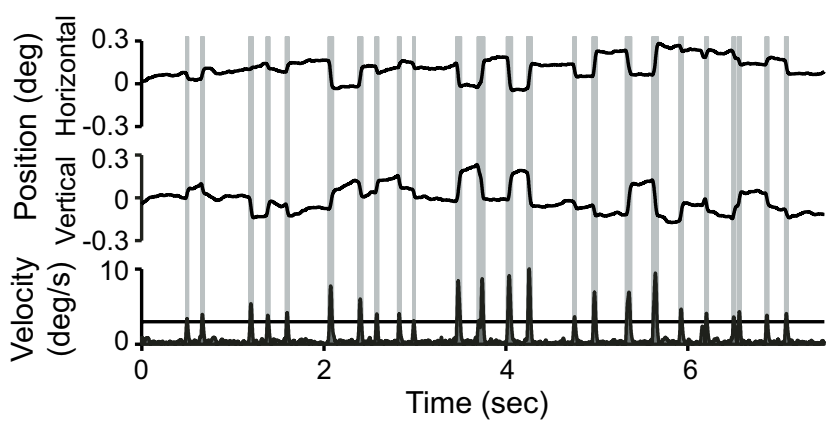

B

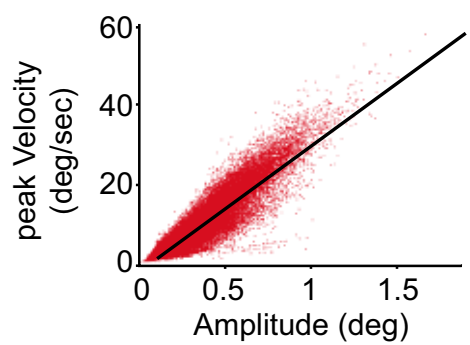

C

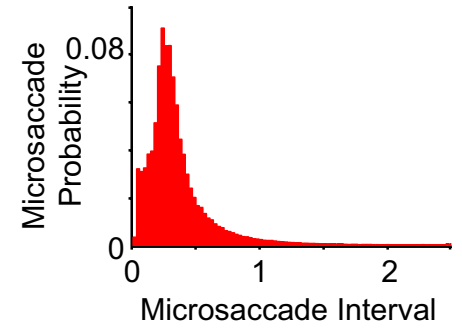

$(\mathrm{sec})$

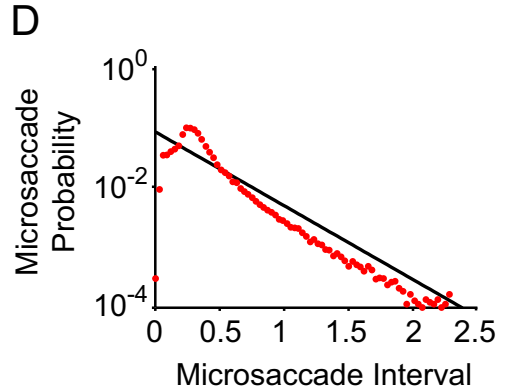

(sec)

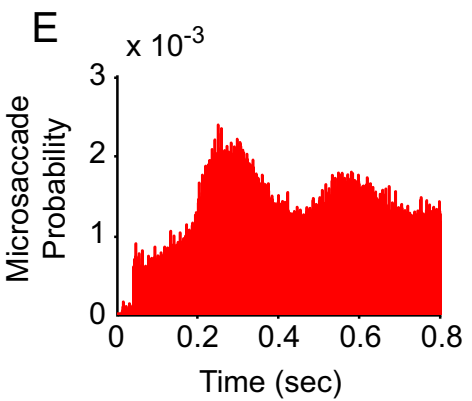

Figure 1. Microsaccade assessment and statistical characterization. $A$, Example of eye movement traces and MS detection. Eye position in the horizontal direction (top graph) and vertical direction (middle graph) and the corresponding eye velocity (bottom graph) as a function of time during fixation. The horizontal line in the velocity plot shows the threshold used to detect MSs. Time periods classified as MSs are shaded in gray. $\boldsymbol{B}$, Scatter plot of MS amplitude versus MS peak velocity (with 1 dot per MS pooled over all MSs used for the neurophysiological analysis), demonstrating the linear relation between both parameters $(r=0.812, p<$ $0.001, t=382$ ). $C$, Histogram (bin width, $0.027 \mathrm{~s}$ ) of MS probability as a function of time since the last MS. This inter-MS interval histogram shows a peak of MS occurrence at $0.25 \mathrm{~s}$, followed by an exponentially decaying tail. $\boldsymbol{D}$, Same data as in C, but in semilogarithmic scale to compare with an exponential distribution. The black line illustrates the best-fitting exponential distribution. $E, M S$ autocorrelation histogram (bin width, $0.001 \mathrm{~s}$ ), directly demonstrating the MS rhythmicity. deg, Degree.

Spectra and cross-spectra are averaged over trials before calculating the coherency as follows:

$$
C_{y x}(f)=\frac{S_{y x}(f)}{\sqrt{S_{x}(f) S_{y}(f)}}
$$

Coherency is a complex quantity. Its absolute value is termed coherence and ranges from 0 to 1 . A coherence value of 1 indicates that the two signals have a constant phase relationship (and amplitude covariation), whereas a value of 0 indicates the absence of any phase relationship.

We also quantified the phase locking of the LFP to the MSs. This analysis is equivalent to calculating the phase locking of the EEG to a stimulus, except that the stimulus is replaced by the MS. The phase locking of the EEG to a stimulus across trials is often quantified as the "intertrial coherence" (Makeig et al., 2002, 2004). We used the same approach and therefore refer to the phase locking of the LFP to the MSs as "inter-MS coherence." In short, the complex Fourier transforms were first normalized per trial (and also per time and frequency) to unit length, and the inter-MS coherence is then the length of the complex average of those unit length Fourier transforms. Like normal coherence, inter-MS coherence assumes a value of 1 for perfect phase locking and a value of 0 for fully random phase relations.

To estimate SEMs for power spectra, coherence spectra, or inter-MS coherence spectra, we used the jackknife method (Efron and Tibshirani, 1993).

Statistics. We wanted to test whether perimicrosaccadic perturbations of neuronal synchronization were significantly different from random fluctuations. To this end, we compared peri-MS epochs to epochs that were not aligned to MSs. For each \pm 0.8 s peri-MS epoch, we took a control epoch from another trial (usually the next one) that was identical in terms of latency after stimulus onset. Trials had different lengths, and if a corresponding epoch could not be found in the next trial, we searched for it in the next trial, and so forth. If no corresponding epoch could be found anywhere in the dataset, the respective peri-MS epoch was discarded. This procedure resulted in two sets of data epochs: One aligned to the MSs and one that was not MS aligned but was otherwise identical. We determined power, coherence, and inter-MS coherence and their SEMs separately for the two sets of epochs and compiled $t$ values for their comparison. This was done independently for each pixel in the time-frequency image. Thus, for each LFP recording, we obtained a time-frequency $t$ image of peri-MS power perturbations and inter-MS coherence. Correspondingly, for each pair of spike and LFP recordings, we obtained a time-frequency $t$ image of peri-MS coherence perturbations.

Next, we evaluated whether $t$ values obtained per (pair of) recording site(s) were also reliable across (pairs of) recording sites. To this end, we tested, for each time-frequency pixel independently, whether this pixel's distribution of $t$ values across (pairs of) recording sites was significantly different from zero, using a one-sample $t$ test.

This latter $t$ test was done for all time-frequency pixels and we therefore corrected for the multiple comparisons, using a nonparametric permutation approach for significance testing (Nichols and Holmes, 2001; Maris and Oostenveld, 2007). To this end, the following procedure was performed 10,000 times: for each recordings site (or pair of recording sites), a random decision was made to multiply the $t$ values obtained previously by -1 ( $50 \%$ of probability) or 1 ( $50 \%$ of probability). Subsequently, a new one-sample $t$ test was performed across the sites (or pairs of sites) separately for all time-frequency pixels. Only the maximal and minimal $t$ value across all time-frequency pixels was kept, resulting in 10,000 minimal and 10,000 maximal $t$ values. From this empirical distribution of maximal and minimal values, we determined the $2.5 \%$ and $97.5 \%$ points, $t_{\text {global, } 2.5 \%}$ and $t_{\text {global, } 97.5 \%}$. Then, we evaluated the observed time-frequency $t$ image against these thresholds. A value was considered as significant if it was larger than $t_{\text {global, } 97.5 \%}$ or smaller than $t_{\text {global, } 2.5 \%}$. This procedure corresponds to a two-sided test with a false-positive rate of $5 \%$ and corrects for the multiple comparisons across time and frequency.

Time-frequency differences of peri-MS coherence perturbations centered at gamma-band activity (see Fig. $4 C$ ) were also used to evaluate the efficiency of visuomotor transformations. To that purpose, we convolve a $\pm 0.3 \mathrm{~s}$ segment of the observed perturbation around the 
MS with the MS rate time courses of the $25 \%$ slowest/fastest trials. Statistical differences were calculated following the same procedure described previously.

The statistical testing for firing rates followed the same procedure, except that there was no frequency dimension involved.

\section{Results}

\section{Identification and temporal}

distribution of microsaccades

Eye movements and V1/V4 data were obtained from two monkeys performing a behavioral task that involved prolonged fixation and visual stimulation [see Materials and Methods for details; the data have been used in several previous publications (Fries et al., 2001, 2008b; Womelsdorf et al., 2006, 2007)]. The present analysis focused on MS-related modulations in neuronal activation and/or synchronization and therefore data were pooled across behavioral conditions. During the task, monkeys fixated their gaze within a $\pm 0.7^{\circ}$ window around a fixation point. Fixational eye movements were considered for the analysis if they did not exceed the limits imposed by this fixation window. Figure $1 A$ shows eye movements during a representative fixation period.

To identify MSs, we evaluated peak velocities and amplitudes of ocular movements (see Materials and Methods). Figure $1 A$ shows a trial in which MSs were identified according to these criteria. To test whether putative MSs conformed to the known ballistic nature of eye movements, we investigated the relation between MS amplitudes and peak velocities. We confirmed a linear relation between both parameters (Fig. $1 B)(r=0.812, p<0.01$, $t=382$ ) (Zuber and Stark, 1965; Bair and O'Keefe, 1998; Martinez-Conde et al., 2000, 2006; Hafed et al., 2009).

Microsaccades occurred with an average rate of more than two times per second, consistent with previous reports (Bair and O’Keefe, 1998; Martinez-Conde et al., 2000, 2002; Engbert and Mergenthaler, 2006). A recent study demonstrated that MSs in human subjects occur with a distinct temporal structure (OteroMillan et al., 2008). To test for a corresponding temporal structure in our monkey data, we compiled an inter-MS interval histogram (Fig. 1C,D), as well as an autocorrelation histogram (Fig. $1 E$ ) of the MS occurrence times. Inter-MS intervals beyond $1 \mathrm{~s}$ obeyed an exponential probability distribution (Fig. $1 D)$, consistent with a Poisson process. However, within a second of the last MS, the likelihood for the next MS is reduced for the first $0.2 \mathrm{~s}$ and is particularly high at $\sim 0.25-0.3 \mathrm{~s}$ (Fig. $1 C, D$ ) (Engbert and Mergenthaler, 2006). Consistent with this observation, the autocorrelation histogram demonstrated a rhythmic MS reoccurrence approximately each $0.25-0.3 \mathrm{~s}$ (Fig. $1 E$ ).

This is consistent with "a periodic component of the generating process" of MSs, as has been concluded previously (Engbert and Mergenthaler, 2006). External rhythmic drive is unlikely in our case. The gratings were drifting, but their temporal frequencies were between one and two cycles/degree in different sessions
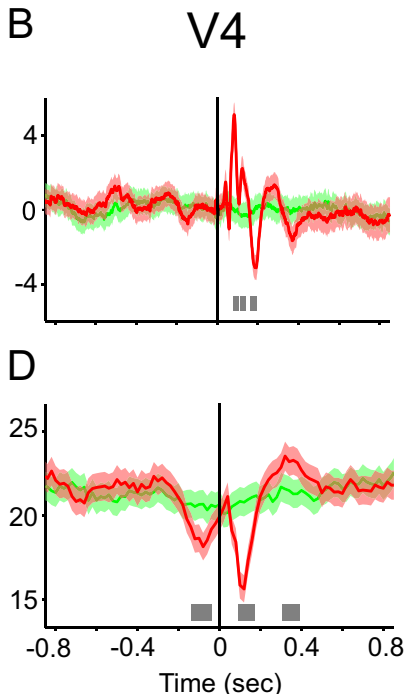

$\mathrm{F}$

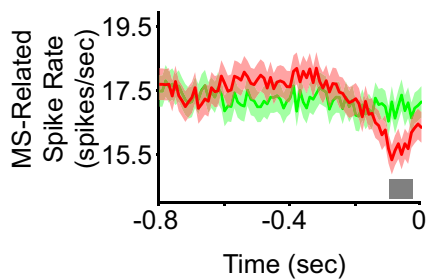

Figure 2. Microsaccade-related modulations in LFP and spike rate in V1 and V4. $A, B, C, D, F$, Comparison between MSs related time course averages across sessions (red lines) and fake MSs time course averages across sessions (green lines) (see Materials and 作 modulation ( $p<0.05$, randomization test, corrected for multiple comparisons). $\boldsymbol{B}$, Same as $A$, but for V4. C, D. Same as $A$ and $B$ function after excluding from the analysis all MSs that were preceded by other MSs within a window of $0.1-0.6 \mathrm{~s}$. The red histogram shows all MSs for comparison. $\boldsymbol{F}$, The same as $\boldsymbol{D}$, but after the MS selection as described for $\boldsymbol{E}$.

(Fries et al., 2001). Also, no signs of optokinetic nystagmus were present in the raw eye position traces (Fig. 1A). We therefore went on to study whether neuronal activity in visual areas V1 and/or V4 showed signs of modulation with respect to MSs.

\section{Perimicrosaccadic modulations of spike rate and local field potential}

To test for modulations in spike rate and/or LFP, time locked to MSs, we compiled respective averages aligned to MS onsets. Local field potentials both in V1 and V4 showed strong phase-locked components during the first $0.4 \mathrm{~s}$ after the MS (Fig. $2 A, B$ ). Spike rates in both V1 and V4 showed rapid post-MS dynamics (Fig. $2 C, D)$. In addition, spike rates in V4 exhibited a significant trough preceding the MS (Fig. 2D).

Previous studies of MS-related spike rate modulation found inconsistent results, which necessarily agree only partly with our results (Leopold and Logothetis, 1998; Martinez-Conde et al., 2000, 2002; Snodderly et al., 2001; Reppas et al., 2002; Kagan et al., 2008). Some of those previous studies reported modulations in firing rate preceding the MS (Leopold and Logothetis, 1998; Martinez-Conde et al., 2000, 2002). We wondered whether the pre-MS modulation of firing rate that we found in V4 could be explained as a consequence of the MS rhythmicity that we described above. If the MS to which our analysis is aligned is preceded by rhythmic MSs, then modulations after those preceding MSs would appear like modulations preceding the MS to which 
A

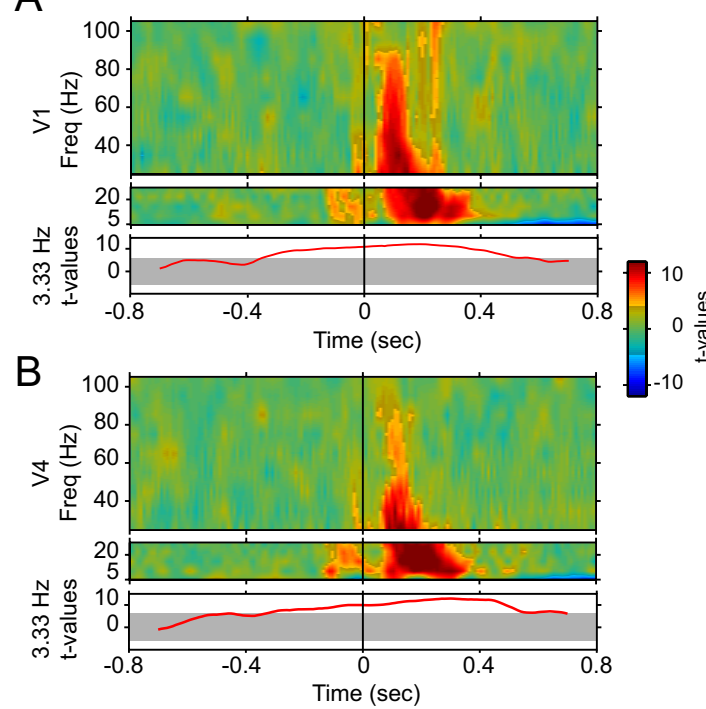

C
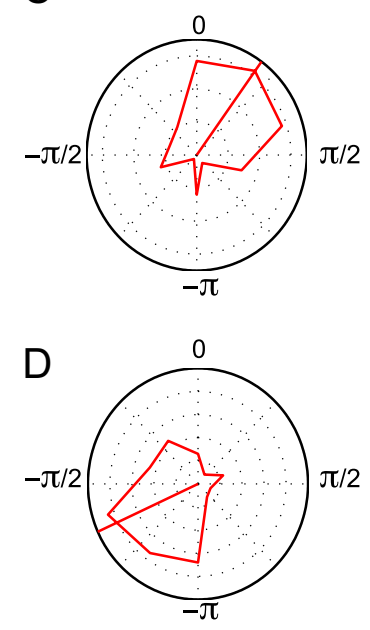

$-0.333 \mathrm{~s}$

before MSs onset

Figure 3. Frequency-wise phase locking of LFP to MSs. $\boldsymbol{A}$, Top, Time-frequency representation of the inter-MS coherence, expressed as $t$ values for the comparison between MS-aligned and non-MS-aligned LFP segments from V1. Results are shown separately for lower and higher frequency ranges, because different spectral concentrations were used (see Materials and Methods). Bright (gray-masked) colors indicate significance (insignificance) of the respective modulations ( $p<0.05$, corrected for multiple comparisons across time and frequency). Bottom, Same analysis restricted to the frequency bin of $3.33 \pm 1.6 \mathrm{~Hz}$. The gray area indicates the significance threshold. $\boldsymbol{B}$, Same as $\boldsymbol{A}$, but for data from V4. C, Polar histogram of V1 LFP phase distributions for $3.33 \pm 1.6 \mathrm{~Hz}$ and for a 0.6 -s-long analysis window centered at $0.333 \mathrm{~s}$ before MS onset. $\boldsymbol{D}$, Same as $\boldsymbol{C}$, but for data from V4.

the analysis was aligned. To control for this possibility, we excluded from the analysis all MSs that were preceded by other MSs within a window of $0.6-0.1 \mathrm{~s}$. This left the pre-MS phasic reduction in firing rate in V4 essentially unchanged (Fig. 2E,F). In Figure 2, $D$ and $F$, the horizontal lines at the bottom illustrate significant modulations ( $p<0.05$, randomization test) and the fact that the control analysis did not change the effect.

\section{MS-locked synchronization}

As has been pointed out previously, MSs might serve to spatially and temporally synchronize firing (Martinez-Conde et al., 2000). Synchronization is most often rhythmic and therefore best described in the frequency domain. Time domain averages, as just considered, lump all frequencies together and might thereby obscure spectrally specific phase consistencies between MSs and neuronal signals. Therefore, we quantified the phase-locking spectrum of the LFP to the MS. This is similar to calculating the phase locking of the EEG to a stimulus across trials, which has been termed "intertrial coherence" (Makeig et al., 2002, 2004). We will therefore refer to the phase locking of the LFP to the MSs as inter-MS coherence.

This analysis demonstrated an MS-related perturbation of the LFP that is phase locked to the MS, starts within the first $0.1 \mathrm{~s}$ after the MS (the precise latency is hard to determine because of the analysis window), and lasts until $\sim 0.4 \mathrm{~s}$ (Fig. $3 A, B$, top). During that time, the dominant frequency components dropped from $\sim 60 \mathrm{~Hz}$ down to $10 \mathrm{~Hz}$ and further (see below and Fig. $3 A, B$, bottom). This inter-MS coherence might underlie the MS-related LFP responses shown in Figure 2, $A$ and $B$. The analysis also revealed a phase-locked component between 10 and $20 \mathrm{~Hz}$, preceding the MS by $\sim 0.1 \mathrm{~s}$. Given the analysis window length of $0.4 \mathrm{~s}$, it cannot be excluded that this is a side effect of the MSrelated LFP response. Yet, this pre-MS effect is temporally dis- continuous with the post-MS effect described above, suggesting that it is not simply because of temporal smearing.

Given the rhythmicity of MSs at $\sim 3.3$ $\mathrm{Hz}$, we also wondered whether the LFP in this frequency band is phase locked to the MS occurrences and particularly whether such a potential phase locking might precede the MS. To this end, we conducted a dedicated inter-MS coherence analysis tailored to this low frequency by using a sliding window of $0.6 \mathrm{~s}$ in length (Fig. $3 A, B$, bottom). In both V1 and V4, we found $3.3 \mathrm{~Hz}$ inter-MS coherence peaking a few hundred milliseconds post-MS. But significant $3.3 \mathrm{~Hz}$ inter-MS coherence started sufficiently early before the MS such that the respective LFP components cannot be evoked by the MS but rather predict the upcoming MS. At $0.33 \mathrm{~s}$ before the MS, when our $0.6 \mathrm{~s}$ analysis window still safely excluded the MS itself, LFP phases at $3.3 \mathrm{~Hz}$ were distributed highly nonuniformly and were thereby predictive of the upcoming MS (Fig. 3C,D).

Importantly, for all analyses of peri-MS modulations in synchronization (whether time locked or not), we compared sliding windows that were aligned to MSs with sliding windows that were not aligned to MSs but identical in their temporal relation to stimulus and task onset (see Materials and Methods for details). This approach ensured that potential effects of time after stimulus/task onset were not mistaken as effects of time relative to the MSs.

\section{Peri-MS modulation of rhythmic synchronization in area V1}

The visual stimulation induced strong ongoing gamma-band synchronization. We evaluated whether MSs modulated this ongoing rhythmic synchronization (regardless of phase locking to the MS) (see Materials and Methods for details). We first investigated LFP power in area V1 and observed several significant perimicrosaccadic modulations (Fig. $4 A, B$ ). First, after the MS, power increased in several frequency bands, namely, $60-100 \mathrm{~Hz}$, $15-40 \mathrm{~Hz}$, and $\sim 10 \mathrm{~Hz}$, with periodic fluctuations in amplitude. Second, the first $0.2 \mathrm{~s}$ after the MS was dominated by a strong reduction in synchronization in the classical gamma-frequency band $(40-60 \mathrm{~Hz})$.

Third, and more tentatively, we found what appeared to be diagonal bands in the time-frequency plots, with bands of enhanced synchronization separated by bands of reduced synchronization (supplemental Fig. S1, available at www.jneurosci.org as supplemental material). Each band extended from high frequencies to low frequencies. This periodic stripe pattern had a frequency of $\sim 3.3 \mathrm{~Hz}(\sim 4$ cycles within $1.2 \mathrm{~s})$ and was aligned to the MS such that the post-MS gamma-band desynchronization was part of it. We had noted above that MSs occurred rhythmically with a frequency of $\sim 3-4 \mathrm{~Hz}$ (Fig. 1C,E). We therefore considered that this stripe pattern might be related to the rhythmic MS reoccurrence, and this possibility is explored in the next section below.

We also found significant coherence between spikes and LFPs in area V1. This spike-field coherence showed a rapid post-MS desynchronization followed by a transient increase, with a diagonal band appearance that seemed similar to that in the LFP (Fig. 

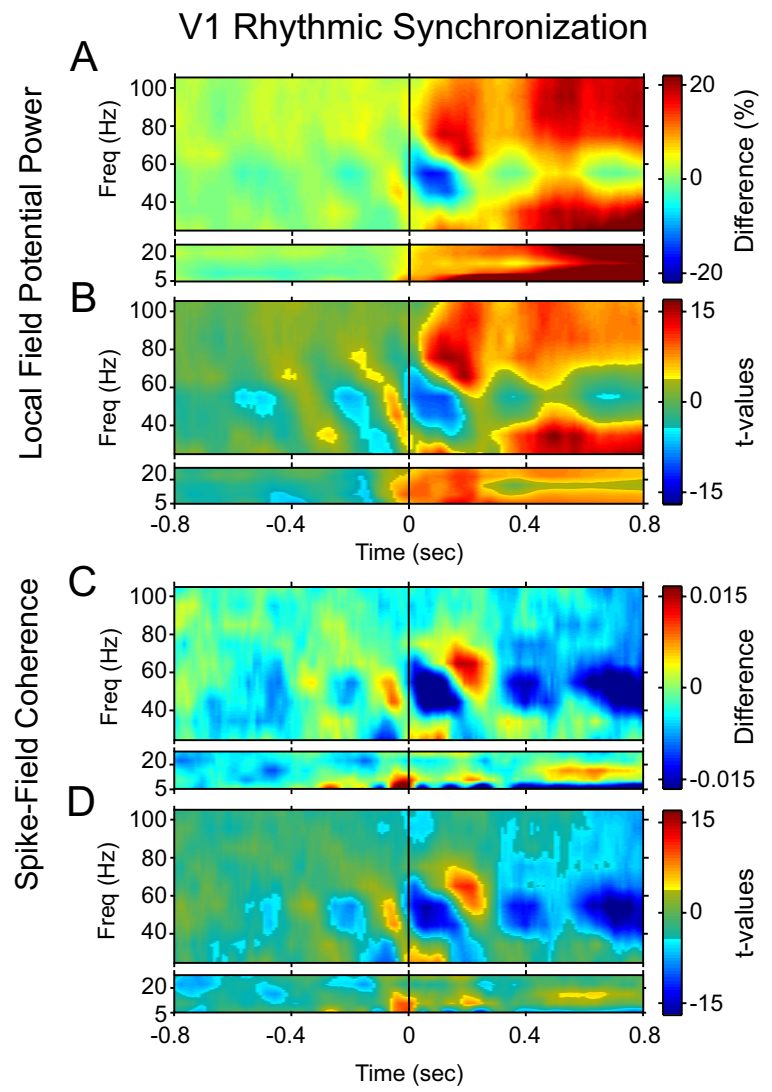

Figure 4. Peri-MS modulation of rhythmic synchronization in V1. $A$, Time-frequency representation of peri-MS modulations in LFP power as a function of time relative to the MS. Results are shown separately for lower and higher frequency ranges, because different spectral concentrations were used (see Materials and Materials and Methods). $\boldsymbol{B}$, Time-frequency representation of corresponding $t$ values. Bright (gray-masked) colors indicate significance (insignificance) of the respective modulations ( $p<0.05$, corrected for multiple comparisons across time and frequency). $\boldsymbol{C}, \boldsymbol{D}$, Same as $\boldsymbol{A}$ and $\boldsymbol{B}$, but for spike-field coherence. Freq, Frequency.

$4 C, D)$. However, not all features of the MS-related perturbation of LFP power were paralleled by perturbations in SFC. Whereas the LFP showed longer-lasting power increases in several frequency bands, spike-field coherence did not. Rather, there was a prolonged decrease in the $40-60 \mathrm{~Hz}$ band.

\section{Nature of pre-MS perturbations of neuronal synchronization in $\mathrm{V} 1$}

We found that, in V1, an MS is typically followed by a reduction of 40-60 Hz LFP power and a subsequent enhancement of $60-$ $100 \mathrm{~Hz}$ power within the first $0.2 \mathrm{~s}$ after the MS. Similar patterns of reduction and enhancement occurred before MSs, starting $\sim 0.2 \mathrm{~s}$ and $0.6 \mathrm{~s}$ pre-MS. These pre-MS perturbations might be triggered by rhythmic MSs, preceding the MS to which the analysis has been aligned (same reasoning as above for the pre-MS firing rate modulation). To test for this, we repeated the analysis, but used reference MSs (and -0.8 to $0 \mathrm{~s}$ of data around them) only if there had been no other MS within a window of $0.6-0.1 \mathrm{~s}$ before the reference MS. The result of this control analysis is shown in Figure $5 A$, demonstrating that eliminating preceding MSs also eliminated the power modulations. Thus, in V1, power modulations preceding the reference MS were most likely actually after preceding MSs (that were rhythmically distributed in time) and therefore did not really precede MSs but only appeared to do so [for a similar analysis, see also the study by MartinezConde et al. (2002)]. Please note that selecting MSs not preceded

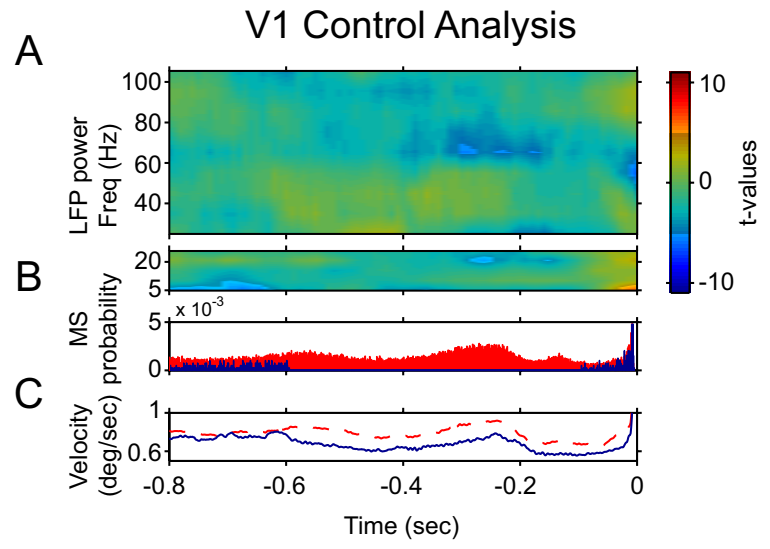

Figure 5. Pre-MS enhancement of V1 gamma-band synchronization is caused by MS rhythmicity. $A$, Same analysis as for Figure $3 B$, but after excluding from the analysis all MSs that were preceded by other MSs within a window of $0.1-0.6 \mathrm{~s}$. Freq, Frequency. $\boldsymbol{B}$, This panel documents the MS selection applied in $\boldsymbol{A}$ and uses only MSs obtained during V1 recordings. The blue histogram shows the MS autocorrelation function after excluding from the analysis all MSs that were preceded by other MSs within a window of -0.6 to $-0.1 \mathrm{~s}$. The red histogram shows this autocorrelation function for all MSs. $\boldsymbol{C}$, The blue line uses the same MSs for the alignment of the analysis as the blue histogram in $\boldsymbol{B}$, but shows the pre-MS eye velocity. The red line shows the same without MS selection.

by other MSs in a certain time window is equivalent to selecting epochs that were unusual in terms of their low-frequency dynamics. The elimination of power modulations could therefore be due either to the elimination of preceding MSs or to the selection of epochs with unusual low-frequency dynamics. We cannot dissociate these two options.

Peri-MS modulation of rhythmic synchronization in area V4 Rhythmic synchronization in V4 showed peri-MS modulations that only partly resembled those in V1 (Fig. 6A). The greatest similarity to V1 was in the early post-MS decrease in $40-60 \mathrm{~Hz}$ LFP power. This feature was slightly slower in onset and slightly more prolonged in V4 compared with V1. The post-MSs power enhancements were also present in V4. However, they had a later onset and the 60-100 Hz enhancement was restricted to a period from 0.3 to $0.5 \mathrm{~s}$. The most prominent difference to $\mathrm{V} 1$ was that LFP power in V4 showed a strong and significant enhancement from 40 to $100 \mathrm{~Hz}$ and from 0.5 to $0.25 \mathrm{~s}$ before the MSs. This enhancement was found very reliably across recording sites (Fig. $7)$. In the next section below, we explore whether this pre-MSs power enhancement is attributable to rhythmically preceding MSs, as had been the case for the pre-MS modulations in V1.

Spike-field coherence (Fig. 6C,D) showed peri-MS modulations similar to the LFP power. The main difference was that the enhancements, both pre- and post-MS, were more restricted in time and frequency extent.

\section{Nature of pre-MS perturbations in neuronal synchronization in $\mathrm{V} 4$}

We found that, in V4, an MS is typically followed by a 60-100 $\mathrm{Hz}$ power enhancement $\sim 0.4$ s post-MS. Correspondingly, the $60-100 \mathrm{~Hz}$ enhancement $\sim 0.4 \mathrm{~s}$ before the MS might be triggered by MSs that occurred $\sim 0.8 \mathrm{~s}$ before the reference MS. To test for this, we repeated the analysis, but used MSs (and -0.8 to $0 \mathrm{~s}$ of data around them) only if there had been no other MS within 1-0.6 s before the reference MS. The result of this control analysis is shown in Figure $8 A$, which demonstrates the same increase in $60-100 \mathrm{~Hz}$ power before the MS as in 


\section{V4 Rhythmic Synchronization}
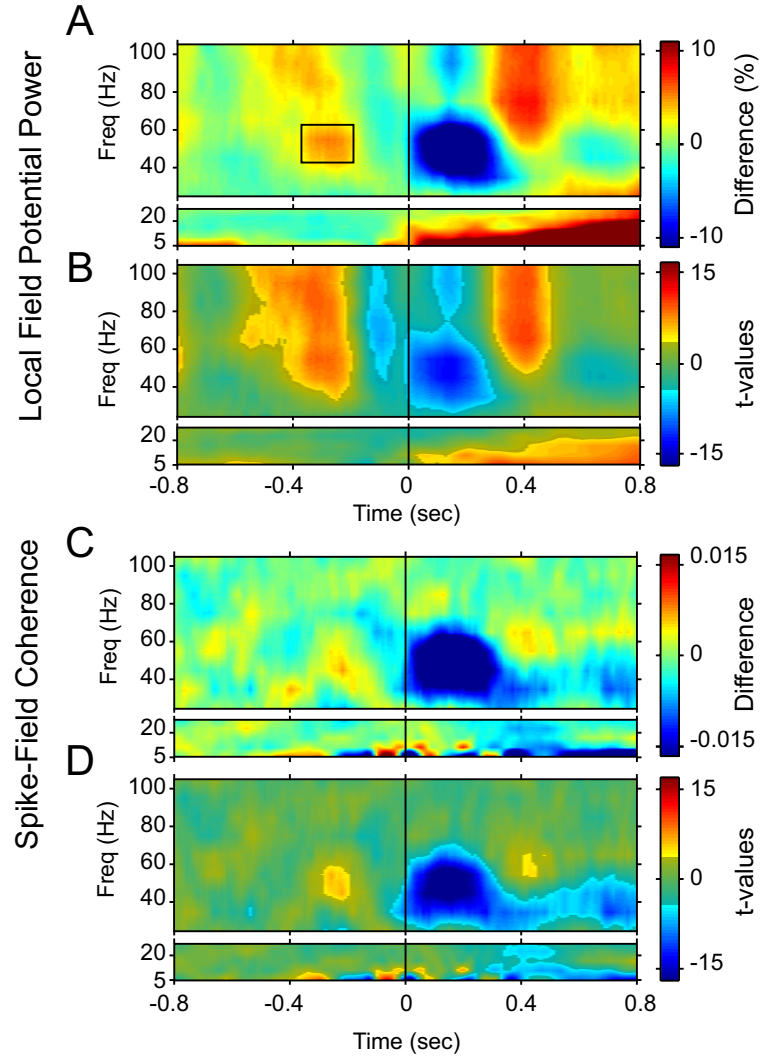

Figure 6. Peri-MS modulation of rhythmic synchronization in V4. A, Time-frequency representation of peri-MS modulations in LFP power as a function of time relative to the MS. Results are shown separately for lower and higher frequency ranges, because different spectral concentrations were used (see Materials and Methods). The black rectangle represents the timefrequency tile used for the analysis shown in Figure 7. B. Time-frequency representation of corresponding $t$ values. Bright (gray-masked) colors indicate significance (insignificance) of the respective modulations ( $p<0.05$, corrected for multiple comparisons across time and frequency). $\boldsymbol{C}, \boldsymbol{D}$, Same as $\boldsymbol{A}$ and $\boldsymbol{B}$, but for spike-field coherence. Freq, Frequency.

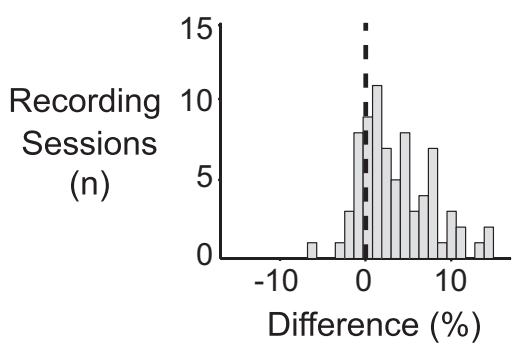

Figure 7. Consistency of pre-MS enhancement of gamma-band synchronization. Histogram of relative change in LFP power across all recording sites for the time-frequency tile indicated in Figure $6 \mathrm{~A}$ (comparing peri-MS periods with periods not aligned to MSs, as explained in Materials and Methods).

Figure 6 . Thus, the enhancement $\sim 0.4 \mathrm{~s}$ pre-MS is most likely not triggered by previous MSs but truly precedes the reference MS. Figure $8 B$ demonstrates that MSs had indeed been eliminated as planned, and Figure $8 C$ shows that this resulted in an almost completely flat eye velocity profile within the controlled time window.

\section{Effects of MS rhythmicity on visuomotor transformation}

The analysis so far has demonstrated that MSs modulate GBS in areas $\mathrm{V} 1$ and V4 with different temporal profiles. We next asked

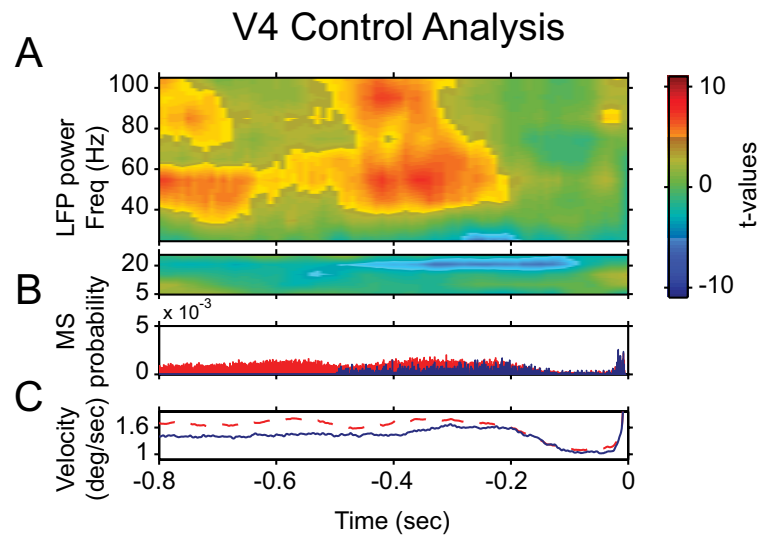

Figure 8. Pre-MS enhancement of V4 gamma-band synchronization is not caused by MS rhythmicity. $\boldsymbol{A}$, Same analysis as for Figure 4, but for data from V4 after excluding from the analysis all MSs that were preceded by other MSs within a window of -1.0 to $-0.5 \mathrm{~s}$. Freq, Frequency. $\boldsymbol{B}$, This panel documents the MS selection applied in $\boldsymbol{A}$ and uses only MSs obtained during V4 recordings. The blue histogram shows the MS autocorrelation function after excluding from the analysis all MSs that were preceded by other MSs within a window of $0.5-1.0 \mathrm{~s}$. The red histogram shows this autocorrelation function for all MSs. $C$, The blue line uses the same MSs for the alignment of the analysis as the blue histogram in $\boldsymbol{B}$ but shows the pre-MS eye velocity. The red line shows the same without MS selection. deg, Degree.

whether the MS-induced modulations in GBS had consequences for the efficiency of visuomotor transformations. It has been shown previously that visuomotor transformations are particularly fast when a behaviorally relevant stimulus change is processed by a visual cortex that is already engaged in particularly precise GBS (Womelsdorf et al., 2006). GBS can fluctuate spontaneously and thus can be high or low at the moment of the randomly timed stimulus change that the monkey has to report (Womelsdorf et al., 2006). We used the behavioral reaction times (RTs) as indicators of the speed of visuomotor transformation and sorted the trials into reaction time quartiles. We compared the trials with the slowest RTs and fastest RTs. Figure $9 B$ shows that the reaction times are slower on trials with more MSs around the time of the target color changes and fewer MSs $\sim 0.15$ s before the changes.

We next asked whether the different MS rate time courses might produce the slower/faster RTs through modulating visual cortical gamma-band synchronization and/or spike activity. To test this, we used the above-described peri-MS modulation of GBS (measured as gamma-band SFC) and the peri-MS modulation of spike rate (Fig. $2 C, D$ ) and convolved it with the MS rate time courses preceding slower/faster behavioral responses. This was done separately for the peri-MS modulations that we had found in area V1 (Fig. 9C,D) and area V4 (Fig. 9E,F). Figure 9C shows that in trials with slow (fast) RTs, the corresponding MS rate time course produced particularly weak (strong) GBS in V1 $\sim 0.1$ s after the stimulus change. Figure $9 D$ shows the same analysis for firing rate modulations and reveals that in trials with slow (fast) RTs, the MS rate time course produced particularly weak (strong) firing in $\mathrm{V} 1$ from $0.15 \mathrm{~s}$ before the stimulus change to $0.2 \mathrm{~s}$ after the stimulus change. The corresponding analyses for V4 are shown in Figure 9, $E$ and $F$. They reveal enhanced GBS for fast RTs peaking $\sim 0.2 \mathrm{~s}$ after the stimulus change and enhanced firing rates for fast RTs peaking $\sim 0.1 \mathrm{~s}$ after the stimulus change.

\section{Discussion}

In summary, we found that MSs occur rhythmically with a frequency of $\sim 3.3 \mathrm{~Hz}$. Consistent with this rhythmic MS occur- 
rence, MSs are partly predicted by the ongoing neuronal activity. In turn, neuronal activity and neuronal synchronization are modulated around individual MSs, both in areas V1 and V4. In both areas, ongoing visual stimulation resulted in pronounced gamma-band synchronization, and MSs were followed by an early decrease and a late increase in gamma-band synchronization. In area V4, we found a reliable increase in gamma-band synchronization preceding the MS by $\sim 0.4 \mathrm{~s}$. This was followed, $\sim 0.2 \mathrm{~s}$ later, by a decrease in the rate of V4 multiunit firing. These pre-MS modulations in neuronal activity were not attributable to MS rhythmicity, because they were still present when only data in which preceding MSs were absent were analyzed. Most interestingly, we found that the ongoing pattern of MSs around the moment of a behaviorally relevant stimulus change predicts the speed with which the stimulus change is behaviorally reported. Fast behavioral responses are preceded by a pattern of MSs that leads to enhanced gamma-band synchronization and enhanced firing rates in $\mathrm{V} 1$ and V4 at periods when those areas process the stimulus change. Together, these findings indicate that an underlying $3.3 \mathrm{~Hz}$ rhythm might influence all other events, i.e., neuronal firing rates, gamma-band synchronization, MSs, and behavioral reaction times (Lakatos et al., 2005; Schroeder and Lakatos, 2009).

Several previous studies investigated peri-MS modulations of neuronal activity (Bair and O'Keefe, 1998; Leopold and Logothetis, 1998; Martinez-Conde et al., 2000, 2002; Snodderly et al., 2001; Reppas et al., 2002; Supèr et al., 2004; Kagan et al., 2008). Some of these studies reported modulations in neuronal activity preceding MSs (Leopold and Logothetis, 1998; Martinez-Conde et al., 2000, 2002), as has also been reported for saccades (Purpura et al., 2003; Rajkai et al., 2008). Possible explanations have been discussed elsewhere (Martinez-Conde et al., 2002). Other studies reported exclusively post-MS modulation. Thus, previous studies agree only partly among each other and with this study. Where there are discrepancies with the present study, this might be explained by the different measures of neuronal activity: whereas all previous studies focused on isolated single units, we investigated MUA, LFP power, and the MUA-LFP coherence.

LFP power and MUA-LFP coherence have previously been shown to reflect aspects of neuronal group activity and dynamics that are sometimes not captured by isolated single-unit recordings (Fries et al., 1997, 2001, 2002; Logothetis et al., 2001; Niessing et al., 2005; Wilke et al., 2006; Womelsdorf et al., 2006). In particular, LFP-based measures are more sensitive to neural activity than action potentials, because they index the synaptic currents that lead to action potentials (Frost, 1967; Mitzdorf, 1987; Schroeder et al., 1995). Both LFP power and MUA-LFP coherence reflect rhythmic synchronization. MUA-LFP coherence assesses rhythmic synchronization directly as the consistency in phase relation between a MUA and an LFP recording (Pesaran et al., 2002; Womelsdorf et al., 2006). The power of the LFP assesses local synchronization only indirectly, but in practice it is often closely related to MUA-LFP coherence for electrodes spaced within a millimeter of each other, as in our data. Whereas in some cases

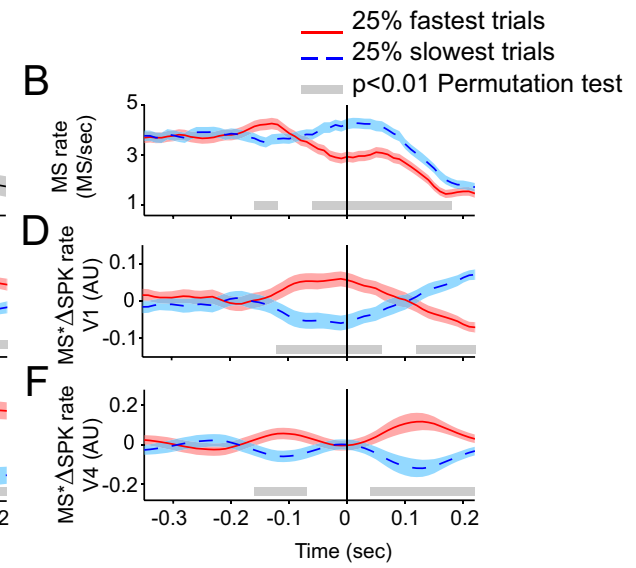

- $25 \%$ fastest trials $\mathrm{p}<0.01$ Permutation tes

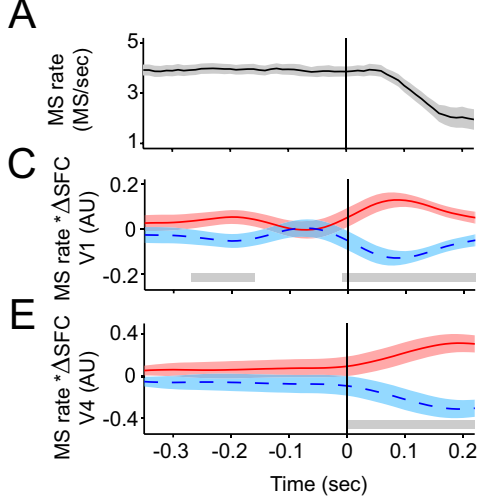

Figure 9. The efficiency of visuomotor transformation is modulated by the MS rhythm and the corresponding modulations in GBS and spike activity. $\boldsymbol{A}$, MS rate as a function of time around stimulus change. MS rate was calculated using a sliding window of $.05 \mathrm{~s}$. Shaded region around the time course represents mean $\pm 95 \%$ confidence interval. $\boldsymbol{B}$, MS rate comparison for trials from (red) and slowest (blue) reaction time quartiles. Bright gray bar at the bottom highlights the significant differences in area V1 (difference between normalized spike rates around MSs compared with equivalent epochs without MSs). $\boldsymbol{E}$, Same as $\boldsymbol{C}$ as $D$ but for data from V4.

rhythmic neuronal synchronization is highly correlated with enhanced firing rates, the two measures are in principle independent, and several reports have demonstrated clear dissociations (Fries et al., 1997, 2001, 2002; Logothetis et al., 2001; Niessing et al., 2005; Wilke et al., 2006; Womelsdorf et al., 2006).

In the period after the MS, we found both in V1 and in V4 an early reduction in gamma-band synchronization followed by a later increase [for a similar gamma power enhancement after large-scale saccades, see Rajkai et al. (2008)]. Both the reduction and the increase occurred earlier in V1 than in V4, consistent with them being a consequence of the MS itself and/or the concurrent retinal shift. The early post-MS suppression of gamma-band synchronization might be related to post-MS elevation of perceptual thresholds (Beeler, 1967), although this psychophysical finding is controversial and the link quite tentative. A closer connection might be made between the later increase in gamma-band synchronization and increases in stimulus visibility. The post-MS increase in gamma-band synchronization in V4 has a latency of $\sim 0.4$ s. Previous studies have demonstrated that the visibility of first- and second-order stimuli is enhanced during this time period after an MS (Martinez-Conde et al., 2006; Troncoso et al., 2008).

Psychophysical effects of MSs were demonstrated in other studies using stimuli that tend to fade from perception, as, e.g., the Troxler stimulus (Martinez-Conde et al., 2006) or artificial scotomas prone to be perceptually filled in (Troncoso et al., 2008). In contrast, we used high-contrast stimuli to induce neuronal activity with high signal-to-noise ratio. These high-contrast stimuli are always visible and therefore do not allow the analysis of variations in visibility. However, they left us the possibility to analyze the speed of behavioral reports of temporally unpredictable stimulus changes. Stimulus changes that were quickly reported were preceded by a temporal pattern of MSs different from that seen for slowly reported changes. The MS pattern that predicted rapid behavioral reports induced strong GBS in V1 $\sim 0.1 \mathrm{~s}$ and in $\mathrm{V} 4 \sim 0.2 \mathrm{~s}$ after the stimulus change. This is intriguing, because the stimulus change is processed in V1 and V4 around those times, and previous studies showed that enhanced 
GBS increases the efficiency of neuronal processing (Womelsdorf et al., 2006). Thus, visual cortical GBS might modulate cortical processing and in turn be modulated by the low-frequency rhythm that controls MSs. The same analysis revealed also a modulation in firing rate with higher firing rates in trials with fast RTs. Those firing rate effects might explain the reaction time differences. The relation between the GBS and the firing rate effects as well as their respective roles in determining the reaction times will require additional investigation.

In this context, we would like to note that MSs are very similar in their rhythmicity to saccades. Figure $1 C$ shows the inter-MS interval histogram and indicates a clear peak at $\sim 0.25$ s. Previous studies demonstrated that intersaccade intervals during free viewing of natural images show very similar distributions (Maldonado et al., 2008; Otero-Millan et al., 2008).

Taking these observations together, we would like to speculate that free-viewing saccades, MSs, and the MS-related modulation of gamma-band synchronization are all related to a general, centrally generated, rhythmic exploration process (Leopold and Logothetis, 1998; Martinez-Conde et al., 2000; Otero-Millan et al., 2008; Hafed et al., 2009). Exploration of visual scenes under natural viewing conditions uses overt saccades as well as microsaccades, which together might provide an optimal discrete sampling of the entire scene (Otero-Millan et al., 2008). Saccades are closely associated with attention, with several studies even suggesting identity of attention and saccade planning (Rizzolatti et al., 1987; Moore and Fallah, 2001; Bisley and Goldberg, 2003). MSs can under certain conditions indicate the allocation of attention (Hafed and Clark, 2002; Engbert and Kliegl, 2003). Consistent with this, microsaccade parameters are modulated by attention and other task demands, e.g., during visual search (Otero-Millan et al., 2008). Attention also modulates gammaband synchronization (Fries et al., 2001; Bichot et al., 2005; Taylor et al., 2005; Bauer et al., 2006; Womelsdorf et al., 2006). Gamma-band synchronization has been shown to be modulated by the phase of lower-frequency rhythms over large parts of the brain (Bragin et al., 1995; Lakatos et al., 2005; Canolty et al., 2006; Osipova et al., 2008; Fries, 2009; Händel and Haarmeier, 2009; Schroeder and Lakatos, 2009). It is striking that these lowerfrequency rhythms can be found back in the temporal patterning of saccadic exploration during free viewing and in the temporal patterning of MSs when overt saccades have to be suppressed. Similar rhythmicity also structures active haptic exploration through whisking in rodents (Fanselow and Nicolelis, 1999; Kleinfeld et al., 1999) and similarities between tactile whisking and fixational eye movements have been suggested (Ahissar and Arieli, 2001; Martinez-Conde and Macknik, 2008). It will be interesting to further elucidate the relation between active exploration, saccades, MSs, and other fixational eye movements on the one hand and, on the other hand, rhythmic neuronal activity in different frequency bands and their mutual interdependencies.

We note a recent study investigating the relation between MSs and power enhancements in the gamma-band range in human scalp EEG recordings (Yuval-Greenberg et al., 2008). Numerous previous studies with human scalp EEG recordings had demonstrated and investigated a broadband power enhancement covering the gamma-band range and occurring $\sim 0.25 \mathrm{~s}$ after the onset of a visual stimulus. Those previous studies had interpreted this broadband power enhancement as a correlate of neuronal gamma-band synchronization. However, the recent study that combined human scalp EEG recordings with MS recordings demonstrated that the broadband power enhancement is not of neuronal origin. Rather, they are electrical artifacts related to the eye muscles that produce MSs. It is important to note that those MS artifacts are unrelated to the MS effects described here. MSrelated artifacts in the EEG are broadband and simultaneous with the MS. In contrast, the effects described here are band limited and precede or follow the MS by many milliseconds (Fries et al., 2008a).

\section{References}

Ahissar E, Arieli A (2001) Figuring space by time. Neuron 32:185-201.

Bair W, O'Keefe LP (1998) The influence of fixational eye movements on the response of neurons in area MT of the macaque. Vis Neurosci 15:779-786.

Bauer M, Oostenveld R, Peeters M, Fries P (2006) Tactile spatial attention enhances gamma-band activity in somatosensory cortex and reduces lowfrequency activity in parieto-occipital areas. J Neurosci 26:490-501.

Beeler GW Jr (1967) Visual threshold changes resulting from spontaneous saccadic eye movements. Vision Res 7:769-775.

Bichot NP, Rossi AF, Desimone R (2005) Parallel and serial neural mechanisms for visual search in macaque area V4. Science 308:529-534.

Bisley JW, Goldberg ME (2003) Neuronal activity in the lateral intraparietal area and spatial attention. Science 299:81-86.

Bragin A, Jandó G, Nádasdy Z, Hetke J, Wise K, Buzsáki G (1995) Gamma $(40-100 \mathrm{~Hz})$ oscillation in the hippocampus of the behaving rat. J Neurosci 15:47-60.

Buzsáki G (2006) Rhythms of the brain. Oxford: Oxford UP.

Canolty RT, Edwards E, Dalal SS, Soltani M, Nagarajan SS, Kirsch HE, Berger MS, Barbaro NM, Knight RT (2006) High gamma power is phaselocked to theta oscillations in human neocortex. Science 313:1626-1628.

Efron B, Tibshirani R (1993) An introduction to the bootstrap. New York: Chapman and Hall.

Engbert R, Kliegl R (2003) Microsaccades uncover the orientation of covert attention. Vision Res 43:1035-1045.

Engbert R, Mergenthaler K (2006) Microsaccades are triggered by low retinal image slip. Proc Natl Acad Sci U S A 103:7192-7197.

Fanselow EE, Nicolelis MA (1999) Behavioral modulation of tactile responses in the rat somatosensory system. J Neurosci 19:7603-7616.

Fries P (2009) Neuronal gamma-band synchronization as a fundamental process in cortical computation. Annu Rev Neurosci 32:209-224.

Fries P, Roelfsema PR, Engel AK, König P, Singer W (1997) Synchronization of oscillatory responses in visual cortex correlates with perception in interocular rivalry. Proc Natl Acad Sci U S A 94:12699-12704.

Fries P, Reynolds JH, Rorie AE, Desimone R (2001) Modulation of oscillatory neuronal synchronization by selective visual attention. Science 291:1560-1563.

Fries P, Schröder JH, Roelfsema PR, Singer W, Engel AK (2002) Oscillatory neuronal synchronization in primary visual cortex as a correlate of stimulus selection. J Neurosci 22:3739-3754.

Fries P, Scheeringa R, Oostenveld R (2008a) Finding gamma. Neuron 58:303-305.

Fries P, Womelsdorf T, Oostenveld R, Desimone R (2008b) The effects of visual stimulation and selective visual attention on rhythmic neuronal synchronization in macaque area V4. J Neurosci 28:4823-4835.

Frost JD (1967) Comparison of intracellular potentials and ECoG activity in isolated cerebral cortex. Electroencephalogr Clin Neurophysiol 23:89-90.

Hafed ZM, Clark JJ (2002) Microsaccades as an overt measure of covert attention shifts. Vision Res 42:2533-2545.

Hafed ZM, Goffart L, Krauzlis RJ (2009) A neural mechanism for microsaccade generation in the primate superior colliculus. Science 323:940-943.

Händel B, Haarmeier T (2009) Cross-frequency coupling of brain oscillations indicates the success in visual motion discrimination. Neuroimage 45:1040-1046.

Jarvis MR, Mitra PP (2001) Sampling properties of the spectrum and coherency of sequences of action potentials. Neural Comput 13:717-749.

Kagan I, Gur M, Snodderly DM (2008) Saccades and drifts differentially modulate neuronal activity in V1: effects of retinal image motion, position, and extraretinal influences. J Vis 8:19:11-25.

Kleinfeld D, Berg RW, O'Connor SM (1999) Anatomical loops and their electrical dynamics in relation to whisking by rat. Somatosens Mot Res 16:69-88.

Kleinfeld D, Ahissar E, Diamond ME (2006) Active sensation: insights from the rodent vibrissa sensorimotor system. Curr Opin Neurobiol 16:435-444. 
Lakatos P, Shah AS, Knuth KH, Ulbert I, Karmos G, Schroeder CE (2005) An oscillatory hierarchy controlling neuronal excitability and stimulus processing in the auditory cortex. J Neurophysiol 94:1904-1911.

Lakatos P, Chen CM, O'Connell MN, Mills A, Schroeder CE (2007) Neuronal oscillations and multisensory interaction in primary auditory cortex. Neuron 53:279-292.

Lakatos P, Karmos G, Mehta AD, Ulbert I, Schroeder CE (2008) Entrainment of neuronal oscillations as a mechanism of attentional selection. Science 320:110-113.

Lee S, Carvell GE, Simons DJ (2008) Motor modulation of afferent somatosensory circuits. Nat Neurosci 11:1430-1438.

Leopold DA, Logothetis NK (1998) Microsaccades differentially modulate neural activity in the striate and extrastriate visual cortex. Exp Brain Res 123:341-345.

Logothetis NK, Pauls J, Augath M, Trinath T, Oeltermann A (2001) Neurophysiological investigation of the basis of the fMRI signal. Nature 412:150-157.

Makeig S, Westerfield M, Jung TP, Enghoff S, Townsend J, Courchesne E, Sejnowski TJ (2002) Dynamic brain sources of visual evoked responses. Science 295:690-694.

Makeig S, Debener S, Onton J, Delorme A (2004) Mining event-related brain dynamics. Trends Cogn Sci 8:204-210.

Maldonado P, Babul C, Singer W, Rodriguez E, Berger D, Grun S (2008) Synchronization of neuronal responses in primary visual cortex of monkeys viewing natural images. J Neurophysiol 100:1523-1532.

Maris E, Oostenveld R (2007) Nonparametric statistical testing of EEG- and MEG-data. J Neurosci Methods 164:177-190.

Martinez-Conde S, Macknik SL (2008) Fixational eye movements across vertebrates: comparative dynamics, physiology, and perception. J Vis 8:28 21-16.

Martinez-Conde S, Macknik SL, Hubel DH (2000) Microsaccadic eye movements and firing of single cells in the striate cortex of macaque monkeys. Nat Neurosci 3:251-258.

Martinez-Conde S, Macknik SL, Hubel DH (2002) The function of bursts of spikes during visual fixation in the awake primate lateral geniculate nucleus and primary visual cortex. Proc Natl Acad Sci USA 99:13920-13925.

Martinez-Conde S, Macknik SL, Hubel DH (2004) The role of fixational eye movements in visual perception. Nat Rev Neurosci 5:229-240.

Martinez-Conde S, Macknik SL, Troncoso XG, Dyar TA (2006) Microsaccades counteract visual fading during fixation. Neuron 49:297-305.

Mehta SB, Whitmer D, Figueroa R, Williams BA, Kleinfeld D (2007) Active spatial perception in the vibrissa scanning sensorimotor system. PLoS Biol 5:e15.

Mitra PP, Pesaran B (1999) Analysis of dynamic brain imaging data. Biophys J 76:691-708.

Mitzdorf U (1987) Properties of the evoked potential generators: current source-density analysis of visually evoked potentials in the cat cortex. Int J Neurosci 33:33-59.

Moore T, Fallah M (2001) Control of eye movements and spatial attention. Proc Natl Acad Sci U S A 98:1273-1276.

Nichols TE, Holmes AP (2002) Nonparametric permutation tests for functional neuroimaging: a primer with examples. Hum Brain Mapp 15:1-25.

Niessing J, Ebisch B, Schmidt KE, Niessing M, Singer W, Galuske RA (2005) Hemodynamic signals correlate tightly with synchronized gamma oscillations. Science 309:948-951.
Osipova D, Hermes D, Jensen O, Rustichini (2008) A gamma power is phase-locked to posterior alpha activity. PLoS ONE 3:e3990.

Otero-Millan J, Troncoso XG, Macknik SL, Serrano-Pedraza I, MartinezConde S (2008) Saccades and microsaccades during visual fixation, exploration, and search: foundations for a common saccadic generator. J Vis 8:21 21-18.

Pesaran B, Pezaris JS, Sahani M, Mitra PP, Andersen RA (2002) Temporal structure in neuronal activity during working memory in macaque parietal cortex. Nat Neurosci 5:805-811.

Purpura KP, Kalik SF, Schiff ND (2003) Analysis of perisaccadic field potentials in the occipitotemporal pathway during active vision. J Neurophysio 90:3455-3478.

Rajkai C, Lakatos P, Chen CM, Pincze Z, Karmos G, Schroeder CE (2008) Transient cortical excitation at the onset of visual fixation. Cereb Cortex 18:200-209.

Reppas JB, Usrey WM, Reid RC (2002) Saccadic eye movements modulate visual responses in the lateral geniculate nucleus. Neuron 35:961-974.

Rizzolatti G, Riggio L, Dascola I, Umiltá C (1987) Reorienting attention across the horizontal and vertical meridians: evidence in favor of a premotor theory of attention. Neuropsychologia 25:31-40.

Schoffelen JM, Oostenveld R, Fries P (2005) Neuronal coherence as a mechanism of effective corticospinal interaction. Science 308:111-113.

Schroeder CE, Lakatos P (2009) Low-frequency neuronal oscillations as instruments of sensory selection. Trends Neurosci 32:9-18.

Schroeder CE, Steinschneider M, Javitt DC, Tenke CE, Givre SJ, Mehta AD, Simpson GV, Arezzo JC, Vaughan HG Jr (1995) Localization of ERP generators and identification of underlying neural processes. Electroencephalogr Clin Neurophysiol Suppl 44:55-75.

Singer W, Gray CM (1995) Visual feature integration and the temporal correlation hypothesis. Annu Rev Neurosci 18:555-586.

Snodderly DM, Kagan I, Gur M (2001) Selective activation of visual cortex neurons by fixational eye movements: implications for neural coding. Vis Neurosci 18:259-277.

Supèr H, van der Togt C, Spekreijse H, Lamme VA (2004) Correspondence of presaccadic activity in the monkey primary visual cortex with saccadic eye movements. Proc Natl Acad Sci U S A 101:3230-3235.

Taylor K, Mandon S, Freiwald WA, Kreiter AK (2005) Coherent oscillatory activity in monkey area $\mathrm{v} 4$ predicts successful allocation of attention. Cereb Cortex 15:1424-1437.

Troncoso XG, Macknik SL, Martinez-Conde S (2008) Microsaccades counteract perceptual filling-in. J Vis 8:15:11-19.

Wilke M, Logothetis NK, Leopold DA (2006) Local field potential reflects perceptual suppression in monkey visual cortex. Proc Natl Acad Sci U S A 103:17507-17512.

Womelsdorf T, Fries P, Mitra PP, Desimone R (2006) Gamma-band synchronization in visual cortex predicts speed of change detection. Nature 439:733-736.

Womelsdorf T, Schoffelen JM, Oostenveld R, Singer W, Desimone R, Engel AK, Fries P (2007) Modulation of neuronal interactions through neuronal synchronization. Science 316:1609-1612.

Yuval-Greenberg S, Tomer O, Keren AS, Nelken I, Deouell LY (2008) Transient induced gamma-band response in EEG as a manifestation of miniature saccades. Neuron 58:429-441.

Zuber BL, Stark L (1965) Microsaccades and the velocity-amplitude relationship for saccadic eye movements. Science 150:1459-1460. 